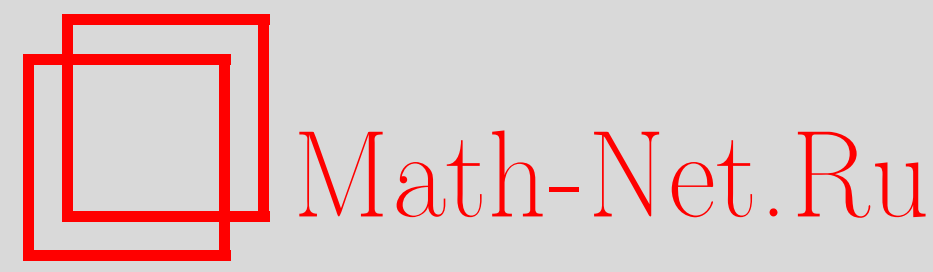

Г. В. Гришина, Об априорных оценках и гладкости решений эллиптической в смысле Дуглиса-Ниренберга системы квазилинейных уравнений, Матем. сб., 1996, том 187, номер 1, 17-40

DOI: https://doi.org/10.4213/sm98

Использование Общероссийского математического портала Math-Net.Ru подразумевает, что вы прочитали и согласны с пользовательским соглашением

http://www.mathnet.ru/rus/agreement

Параметры загрузки:

IP : 54.80 .73 .141

26 апреля 2023 г., 16:57:39 
УДК 517.956.2

\author{
Г.В. Гришина
}

\title{
Об априорных оценках и гладкости решений эллиптической в смысле Дуглиса-Ниренберга системы квазилинейных уравнений
}

\begin{abstract}
Изучается эллиптическая по Дуглису-Ниренбергу система квазилинейных уравнений. Решается задача о предельно допустимом росте нелинейных членов системы относительно их аргументов, при котором возможно получение оценок производных решения через максимум его модуля. Условия на гладкость нелинейных членов минимальные. Результаты точны. Построен пример, показывающий неулучшаемость верхней границы для показателей роста. Априорные $L_{p}$-оценки решения установлены как внутри области, так и вплоть до границы, когда решение удовлетворяет нелинейньм граничньм условиям типа Лопатинского. Исследуется вопрос о гладкости внутри области решений, принадлежащих некоторьм пространствам Соболева. Получены оценки норм Гёльдера производных решения. Доказана теорема об устранимой точечной особенности ограниченных решений общих эллиптических систем квазилинейных уравнений. Bсе результаты являются новьпи и для одного уравнения второго порядка.
\end{abstract}

Библиография: 18 названий.

\section{Введение}

Работа посвящена изучению эллиптических по Дуглису-Ниренбергу систем квазилинейных дифференциальных уравнений. Устанавливаются оценки производных решения через максимум его модуля, и исследуется вопрос о гладкости решений.

Как известно, оценить производные решения через максимум его модуля можно лишь при некоторых ограничениях на рост нелинейных членов уравнения относительно производных неизвестного решения. Так С.Н. Бернштейн показал [1], что для обькновенного дифференциального уравнения

$$
y^{\prime \prime}=f\left(x, y, y^{\prime}\right)
$$

с непрерывной правой частью оценка производной решения через максимум его модуля

$$
\left|y^{\prime}\right| \leqslant C(M), \quad M=\max |y(x)|,
$$

имеет место при выполнении условия

$$
|f(x, y, p)| \leqslant A p^{2}+B,
$$

где $A, B$ - положительные постоянные, которые могут зависеть от $\max |y(x)|$. Показатель 2 в правой части (0.3) является точным. Аналогичњый результат доказан для квазилинейных эллиптических уравнений второго порядка [1], [2]. 
Заметим, что для систем уравнений второго порядка вида (0.1) условие (0.3) не является достаточным для справедливости оценки (0.2). Действительно, функции $y_{1}=\cos \lambda x, y_{2}=\sin \lambda x-$ решения системы

$$
\left\{\begin{array}{l}
\ddot{y}_{1}=-y_{1}\left(\dot{y}_{1}^{2}+\dot{y}_{2}^{2}\right) \\
\ddot{y}_{2}=-y_{2}\left(\dot{y}_{1}^{2}+\dot{y}_{2}^{2}\right)
\end{array}\right.
$$

при любом $\lambda \in \mathbb{R}$, однако оценки вида (0.2) нет.

Оценкам производных решений эллиптических уравнений посвяшено много работ. В монографии О. А. Ладыженской и Н. Н. Уральцевой [3] изложены современные результаты для квазилинейных эллиптических уравнений второго порядка. Уравнениям высшего порядка посвящена монография И. В. Скрыпника [4].

Случай одного квазилинейного уравнения произвольного порядка

$$
\sum_{|\alpha|=2 m} a_{\alpha}(x) D^{\alpha} u(x)=f\left(x, u, D u, \ldots, D^{\alpha} u\right)
$$

подробно изучен С. И. Похожаевым [5] при выполнении неравенства

$$
\left|f\left(x, \xi_{0}, \xi_{1}, \ldots, \xi_{2 m-1}\right)\right| \leqslant b_{0}\left(x, \xi_{0}, \ldots, \xi_{l}\right)+\sum_{k=l+1}^{2 m-1} b_{k}\left(x, \xi_{0}, \ldots, \xi_{l}\right)\left|\xi_{k}\right|^{\nu_{k}}
$$

с положительными функциями $b_{0}, b_{l+1}, \ldots, b_{2 m-1}$, удовлетворяющими условию Каратеодори и принадлежащими некоторым пространствам $L_{p_{k}}, k=0, l+1$, $\ldots, 2 m-1$. В предельном случае, когда $l=0$, и все $p_{k}=\infty$, условие $(0.5)$ принимает вид

$$
\left|f\left(x, \xi_{0}, \xi_{1}, \ldots, \xi_{2 m-1}\right)\right| \leqslant C\left(x, \xi_{0}\right) \sum_{j=1}^{2 m-1}\left(1+\left|\xi_{j}\right|^{2 m / j-\varepsilon}\right),
$$

где $C\left(x, \xi_{0}\right)$ - ограниченная функция, удовлетворяюшая условию Каратеодори, $\varepsilon>0$. Для решений, которые удовлетворяют линейным граничньм условиям на всей границе, им доказана $L_{p}$-оценка производных решения во всей области через максимум его модуля.

Близкие вопросы рассматривались также С. А. Аракчеевым [6]. Для уравнения (0.4) при выполнении условия (0.6) И.В. Скрыпник получил оценку младших производных решения в произвольной внутренней подобласти через максимум его модуля во всей области [4], [7].

В настоящей работе изучается система уравнений вида

$$
\sum_{j=1}^{N} l_{i j}(x, D) u_{j}(x)=f_{i}\left(x, D^{\gamma} u_{j}(x)\right)_{|\gamma| \leqslant s_{i}+t_{j}-1, j=1, \ldots, N}, \quad i=1, \ldots, N,
$$

где $l_{i j}(x, D)$ - линейные дифференциальные операторы в ограниченной области $\Omega \subset \mathbb{R}^{n}$. Изучается вопрос о предельно допустимом росте правых частей относительно производных искомого решения, при котором можно получить априорные 
оценки младших производных решения во внутренних подобластях $\Omega^{\prime}$ области $\Omega$ через максимум его модуля во всей области $\Omega$.

Для уравнения (0.4) это возможно, если $f$ - измеримая по Борелю функция, и

$$
\left|f\left(x, \xi_{0}, \xi_{1}, \ldots, \xi_{2 m-1}\right)\right| \leqslant C\left(x, \xi_{0}\right)\left(1+F\left(\sum_{k=1}^{2 m-1}\left|\xi_{k}\right|^{2 m / k}\right)\right)
$$

где $C\left(x, \xi_{0}\right), F(t)$ - ограниченные, измеримые по Борелю функции, и $F(t)=o(t)$ при $t \rightarrow \infty$. Построен пример, показывающий, что $o(t)$ не может быть заменено на $O(t)$. Для решений системы $(0.7)$ получены $L_{p}$-оценки производных решения как внутри области, так и вплоть до границы, а также внутренние оценки норм Гёльдера младших производных решения через максимум его модуля во всей области.

Предполагаем, что линейные дифференциальные операторы $l_{i j}(x, D)$, входяшие в систему уравнений (0.7), имеют порядок не выше, чем $s_{i}+t_{j}$, где системы целых чисел $\left\{s_{i}\right\}_{i=1}^{N},\left\{t_{j}\right\}_{j=1}^{N}$, таковы, что $s_{i} \leqslant 0,0 \leqslant t_{j} \leqslant t^{\prime}=\max _{j} t_{j}$; $\sum_{i=1}^{N}\left(s_{i}+t_{i}\right)=2 m$. Причем считаем, что $l_{i j} \equiv 0$, если $s_{i}+t_{j}<0$. Коэффициенты $a_{\beta}^{i j}(x),|\beta|=0, \ldots, s_{i}+t_{j},-$ комплекснозначные функции. Пусть $l_{i j}^{\prime}(x, D)$ - оператор, содержащий только члены порядка $s_{i}+t_{j}$ оператора $l_{i j}(x, D)$. Рассмотрим его характеристический многочлен $l_{i j}^{\prime}(x, \xi)$, где $\xi=\left(\xi_{1}, \ldots, \xi_{n}\right)$ - произвольный вектор из $\mathbb{R}^{n}$ и предположим, что

$$
L(x, \xi)=\operatorname{det}\left(l_{i j}^{\prime}(x, \xi)\right)
$$

является многочленом порядка $2 m$, и для любого действительного $\xi \in \mathbb{R}^{n}$ и для любой точки $x \in \bar{\Omega}$ :

$$
A^{-1}|\xi|^{2 m} \leqslant|L(x, \xi)| \leqslant A|\xi|^{2 m}, \quad A=\text { Const }>0
$$

На части $\Gamma \in C^{t^{\prime}}$ границы $\partial \Omega$ области $\Omega$ могут быть заданы краевые условия

$$
\sum_{j=1}^{N} B_{h j}(x, D) u_{j}(x)=\varphi_{h}\left(x, D^{\sigma} u_{j}(x)\right)_{|\sigma| \leqslant r_{h}+t_{j}-1, j=1, \ldots, N}, \quad h=1, \ldots, m
$$

где $B_{h j}(x, D)$ - линейные дифференциальные операторы порядка не выше, чем $r_{h}+t_{j}$ с комплексными коэффициентами $b_{\tau}^{h j}(x),|\tau|=0, \ldots, r_{h}+t_{j}, 1 \leqslant j \leqslant N$, $1 \leqslant h \leqslant m$, где число $r_{h}$ соответствует $h$-му граничному условию. При этом считаем, что $r_{h} \leqslant \max _{i} s_{i}-1$ и $B_{i j} \equiv 0$ при $r_{h}+t_{j}<0$. Предполагаем, что граничные операторы удовлетворяют условиям дополнительности по отношению к системе (0.7) [8], [9].

В дальнейшем относительно правых частей в $(0.7),(0.8)$ предполагаем следуюшее: для каждого $i=1, \ldots, N$ функция $f_{i}\left(x, \xi_{0}^{j}, \ldots, \xi_{s_{i}+t_{j}-1}^{j}\right)_{j=1, \ldots, N}$, где $\xi_{k}^{j}=\left\{\xi_{\beta}^{j}:|\beta|=k\right\}$, определенная на

$$
\Omega \times \mathbb{R}^{N} \times \mathbb{R}^{N_{1}^{1}} \times \cdots \times \mathbb{R}^{N_{s_{i}+t_{1}-1}^{1}} \times \cdots \times \mathbb{R}^{N_{1}^{N}} \times \cdots \times \mathbb{R}^{N_{s_{i}+t_{N}-1}^{N}}
$$


с соответствуюшими $N_{k}^{l}$ со значениями в $\mathbb{R}$, является измеримой по Борелю, если $s_{i}=0$, и имеет непрерывные производные до порядка $-s_{i}$, если $s_{i}<0$. Аналогично, $\varphi_{h}\left(x, \xi_{0}^{j}, \ldots, \xi_{r_{h}+t_{j}-1}^{j}\right)_{j=1, \ldots, N}, h=1, \ldots, m,-$ измеримые по Борелю функции, если $r_{h}=0$, и имеют непрерывные производные до порядка $-r_{h}$, если $r_{h}<0$.

Будем использовать следующие функциональные пространства:

$L_{\infty}(\Omega)$ - пространство измеримых на $\Omega$ функций с нормой

$$
\left\|u ; L_{\infty}(\Omega)\right\|=\operatorname{ess} \max _{x \in \Omega}|u(x)|
$$

$W_{p}^{k}(\Omega)$ - пространство Соболева с нормой

$$
\left\|u ; W_{p}^{k}(\Omega)\right\|=\left(\int_{\Omega} \sum_{|\alpha| \leqslant k}\left|D^{m} u(x)\right|^{p} d x\right)^{1 / p} ;
$$

$W_{p}^{k-1 / p}(\partial \Omega), p>1,-$ пространство функций $\varphi$, определенных на границе $\partial \Omega$ и являющихся граничными значениями функций $v$, принадлежащих $W_{p}^{k}(\Omega)$ с нормой

$$
\left\|\varphi ; W_{p}^{k-1 / p}(\partial \Omega)\right\|=\inf _{v}\left\|v ; W_{p}^{k}(\Omega)\right\|
$$

где нижняя грань берется по всем функциям $v(x)$ из $W_{p}^{k}(\Omega)$, равным $\varphi$ на границе;

$C^{k, \delta}(\Omega), \delta \in(0,1),-$ пространство функций, непрерьвно дифференцируемых в $\Omega$ до порядка $k$, производные порядка $k$ которых удовлетворяют условию Гёльдера с показателем $\delta$, с нормой

$$
\left\|u ; C^{k, \delta}(\Omega)\right\|=\sum_{|\alpha| \leqslant k} \max _{x \in \Omega}\left|D^{\alpha} u(x)\right|+\sum_{|\alpha|=k} \sup _{\substack{x, y \in \Omega \\ x \neq y}} \frac{\left|D^{\alpha} u(x)-D^{\alpha} u(y)\right|}{|x-y|^{\delta}} .
$$

Рассматриваются решения системы $(0.7) u_{j} \in W_{p}^{t_{j}}(\Omega, \operatorname{loc}) \cap L_{\infty}(\Omega), j=1, \ldots, N$. Доказано, что оценку $\left\|u_{j} ; W_{p}^{t_{j}}\left(\Omega^{\prime}\right)\right\|$ через $\left\|u ; L_{\infty}(\Omega)\right\|$ для любой строго внутренней подобласти $\Omega^{\prime}$ области $\Omega$ можно получить, если выполнено условие

$$
\begin{aligned}
\sum_{i=1}^{N} \sum_{0 \leqslant|\alpha| \leqslant-s_{i}} \mid D_{x}^{\alpha} f_{i}(x, & \left.\xi_{0}^{j}(x), \ldots, \xi_{s_{i}+t_{j}-1}^{j}(x)\right)_{j=1, \ldots, N} \mid \\
& \leqslant C\left(x, \xi_{0}^{1}, \ldots, \xi_{0}^{N}\right)\left(1+F\left(\sum_{j=1}^{N} \sum_{k=0}^{t_{j}-1}\left|\xi_{k}^{j}\right|^{t_{j} / k}\right)\right),
\end{aligned}
$$

где $C\left(x, \xi_{0}^{1}, \ldots, \xi_{0}^{N}\right), F(t)$ - ограниченные измеримые по Борелю функции, и $F(t)=o(t)$ при $t \rightarrow \infty$. При аналогичных условиях на рост $\varphi_{h}$ в $(0.8)$ удается получить априорную $L_{p}$-оценку решения вплоть до границы. Доказательство $L_{p}$-оценки усложняется по сравнению с доказательствами в статье [5], так как мы не требуем выполнения граничных условий на всей границе $\partial \Omega$.

Получены результаты, уточняющие теоремы И.В. Скрыпника о гладкости обобшенных решений. А именно, доказана принадлежность решений $u_{j}$ 
системы (0.7) с коэффициентами $a_{\beta}^{i j} \in C^{-s_{i}, \sigma}(\Omega)$, где $\sigma \in(0,1)$, пространству $C^{t_{j}-1, \delta}(\Omega) \quad \forall \delta \in(0,1), j=1, \ldots, N$, и установлены оценки решения в норме $\left\|\cdot ; C^{t_{j}-1, \delta}\left(\Omega^{\prime}\right)\right\|$ через $\left\|u ; L_{\infty}(\Omega)\right\|$, если в условии $(0.9) F(t)=O\left(t^{1-\varepsilon}\right), \varepsilon>0$, при $t \rightarrow+\infty$ или $p t_{j}>n$, и $F(t)=o(t)$ при $t \rightarrow+\infty$. Причем выполнение условия $p t_{j}>n$ влечет принадлежность решений $u_{j}$ пространствам $C^{t_{j}-1, \delta}(\Omega, \operatorname{loc})$, $j=1, \ldots, N$, даже если $F(t)=O(t)$ при $t \rightarrow+\infty$. Однако, как показьвает построенный пример, оценку производных решения в этом случае установить нельзя.

Доказательства основаны на применении известных $L_{p}$-оценок для линейных уравнений [9] и интерполяционных неравенств Гальярдо-Ниренберга [10], [11].

Рассматривается также вопрос об устранимости точечной особенности ограниченных решений эллиптических систем квазилинейных уравнений. Эта проблема изучалась многими авторами. Наиболее точньй результат был получен В. Г. Мазья [12] для одного квазилинейного эллиптического уравнения произвольного порядка в дивергентной форме. Мы рассматриваем, в отличии от В. Г. Мазья, эллиптическую систему квазилинейных уравнений недивергентного вида и доказываем теорему о гладкости ограниченных решений из некоторых пространств Соболева вплоть до точечной особенности.

Автор благодарит В.А. Кондратьева за постановку задачи и полезные обсуждения.

\section{§1. Интерполяционные неравенства}

Этот параграф̆ носит вспомогательный характер. Важную роль при доказательстве изложенных результатов будут играть интерполяционные неравенства Гальярдо-Ниренберга [10], [11] и некоторые следствия из них.

Лемма 1.1 [10], [11]. Пусть $G$ - область из $\mathbb{R}^{n}$, и функиия $v$ принадлежсит пространству $L_{q}(D) \cap W_{r}^{h}(G), 1 \leqslant q, r \leqslant \infty$. Тогда для любого иелого $0 \leqslant j<h$ функция $D_{j} v$ суммируема со степенью $p$, и справедливо неравенстBo

$$
\left\|D^{j} v ; L_{p}(G)\right\| \leqslant C_{1}\left\|D^{h} v ; L_{r}(G)\right\|^{\theta}\left\|v ; L_{q}(G)\right\|^{1-\theta}+C_{2}\left\|v ; L_{q}(G)\right\|
$$

где $р, \theta$ удовлетворяют условиям

$$
\frac{1}{p}=\frac{j}{n}+\theta\left(\frac{1}{r}-\frac{h}{n}\right)+(1-\theta) \frac{1}{q}, \quad \frac{j}{h} \leqslant \theta<1,
$$

а $C_{1}, C_{2}$ - полохсительные постоянные, причем $C_{2}$ зависит от области $G$.

В дальнейшем через $\Sigma_{\rho}$ будем обозначать шар или полушар радиуса $\rho$ в пространстве $\mathbb{R}^{n}$, а через $\mathscr{F}_{\rho}$ - плоскую часть границы полушара $\Sigma_{\rho}$.

ЗАмечАнИЕ. Пусть $G=\Sigma_{1}$ и в неравенстве $(1.1)$ постоянная $C_{2}\left(\Sigma_{1}\right)=K_{1}$. Тогда, если $G=\Sigma_{\rho}$, то

$$
C_{2}\left(\Sigma_{\rho}\right)=K_{1} \rho^{-n / q+n / p-j}, \quad C_{1}\left(\Sigma_{\rho}\right)=C_{1}\left(\Sigma_{1}\right)
$$


СлеДСТВИЕ. Пусть $q=r, \theta=j / h$. Тогда $p=q=r, u$, следовательно, для $D=\Sigma_{\rho}$ неравенство (1.1) принимает вид

$$
\begin{aligned}
\left\|D^{j} v ; L_{p}\left(\Sigma_{\rho}\right)\right\| \leqslant C_{1}\left\|D^{h} v ; L_{r}\left(\Sigma_{\rho}\right)\right\|^{j / h}\left\|v ; L_{q}\left(\Sigma_{\rho}\right)\right\|^{1-j / h} \\
+K_{1} \rho^{-j}\left\|v ; L_{q}\left(\Sigma_{\rho}\right)\right\| .
\end{aligned}
$$

ОпРЕДЕЛЕНИЕ. Через $W_{p}^{h}\left(\Sigma_{R} \cup \mathscr{F}_{R}\right.$, loc), будем обозначать множество функций $v \in W_{p}^{h}(G)$ для любой области $G \subset \Sigma_{R}$ такой, что $\partial G \cap\left\{\partial \Sigma_{R} \backslash \mathscr{F}_{R}\right\}=\varnothing$.

Для функций $v \in W_{p}^{j}\left(\Sigma_{R}\right.$, loc $)$, если $\Sigma_{R}$ - шар, и $v \in W_{p}^{j}\left(\Sigma_{R} \cup \mathscr{F}_{R}\right.$, loc $)$, если $\Sigma_{R}$-полушар, определим

$$
\tilde{\|} D^{j} v\left\|_{t}=\sup _{0<d<R} d^{j+t}\right\| D^{j} v ; L_{p}\left(\Sigma_{R-d}\right) \|,
$$

где $t \geqslant 0$.

ЛЕмма 1.2. Пусть функиия и принадлежит $W_{p}^{h}\left(\Sigma_{R}, \mathrm{loc}\right) \cap L_{p}\left(\Sigma_{R}\right)$ или $W_{p}^{h}\left(\Sigma_{R} \cup \mathscr{F}_{R}, \mathrm{loc}\right) \cap L_{p}\left(\Sigma_{R}\right)$. Тогда существует положительная постоянная $K_{0}$, зависящая от $h, n, p$, такая, что для любого челого $0 \leqslant j<h$ выполнено неравенство

$$
\tilde{\|} D^{j} v\left\|_{t} \leqslant K_{0} R^{t(1-j / h)} \tilde{\|} D^{h} v\right\|_{t}^{j / h}\left\|v ; L_{p}\left(\Sigma_{R}\right)\right\|^{1-j / h}+K_{0} R^{t}\left\|v ; L_{p}\left(\Sigma_{R}\right)\right\| .
$$

СЛЕДСТВИЕ. Для любого $\varepsilon>0$ и для любого иелого $0 \leqslant j<h$ существует положительная постоянная $K^{\prime}$, зависящая от $h, n, p$, такая, что справедливо неравенство

$$
\tilde{\|} D^{j} v\left\|_{t} \leqslant K^{\prime} \varepsilon \tilde{\|} D^{h} v\right\|_{t}+K^{\prime}\left(1+\varepsilon^{\frac{j / h}{j / h-1}}\right) R^{t}\left\|v ; L_{p}\left(\Sigma_{R}\right)\right\|
$$

ДокаЗАТЕЛЬСтво ЛЕммы 1.2. Зафиксируем $d \in(0, R)$, для которого

$$
\tilde{\|} D^{j} v\left\|_{t} \leqslant 2 d^{j+t}\right\| D^{j} v ; L_{p}\left(\Sigma_{R-d}\right) \| .
$$

Покроем $\Sigma_{R-d}$ конечным числом шаров или полушаров $\Xi_{i}$ радиуса $d / 2$ с центром в $\Sigma_{R-d}$, если $\Sigma_{R-d}-$ шар или полушар, соответственно. Применим к функции $v$ в $\Xi_{i}$ неравенство (1.2) и возведем обе его части в степень $p$ :

$$
\int_{\Xi_{i}}\left|D^{j} v\right|^{p} d x \leqslant C_{2}\left(\int_{\Xi_{i}}\left|D^{h} v\right|^{p} d x\right)^{j / h}\left(\int_{\Xi_{i}}|v|^{p} d x\right)^{1-j / h}+C_{3} d^{-p j} \int_{\Xi_{i}}|v|^{p} d x .
$$

Теперь проведем суммирование по $i$ и воспользуемся неравенством Гёльдера в следуюшей форме

$$
\sum_{i} A_{i} B_{i} \leqslant\left(\sum_{i} A_{i}^{r}\right)^{1 / r}\left(\sum_{i} B_{i}^{s}\right)^{1 / s}, \quad \frac{1}{r}+\frac{1}{s}=1,
$$


положив $1 / r=j / h, 1 / s=1-j / h$,

$$
A_{i}=\left(\int_{\Xi_{i}}\left|D^{h} v\right|^{p} d x\right)^{j / h}, \quad B_{i}=\left(\int_{\Xi_{i}}|v|^{p} d x\right)^{1-j / h} .
$$

Получим

$$
\begin{aligned}
& \int_{\Sigma_{R-d}}\left|D^{j} v\right|^{p} d x \\
& \leqslant C_{4}\left(\int_{\Sigma_{R-d / 2}}\left|D^{h} v\right|^{p} d x\right)^{j / h}\left(\int_{\Sigma_{R-d / 2}}|v|^{p} d x\right)^{1-j / h}+C_{5} d^{-p j} \int_{\Sigma_{R-d / 2}}|v|^{p} d x .
\end{aligned}
$$

Возведем обе части последнего неравенства в степень $1 / p$ и применим неравенство $\left(\sum_{k} B_{k}^{p}\right)^{1 / p} \leqslant \sum_{k} B_{k}$ для $B_{k} \geqslant 0, p \geqslant 0$. Умножая затем обе части на $d^{t+j}$, получим

$$
\begin{aligned}
& d^{t+j}\left(\int_{\Sigma_{R-d}}\left|D^{j} v\right|^{p} d x\right)^{1 / p} \\
& \leqslant C_{4}^{1 / p} d^{(h+t) j / h+(1-j / h) t}\left(\int_{\Sigma_{R-d / 2}}\left|D^{h} v\right|^{p} d x\right)^{j / h p}\left(\int_{\Sigma_{R-d / 2}}|v|^{p} d x\right)^{1 / p(1-j / h)} \\
& \quad+C_{5} d^{t}\left(\int_{\Sigma_{R-d / 2}}|v|^{p} d x\right)^{1 / p} \\
& \leqslant C_{4}^{1 / p} R^{t(1-j / h)}\left\|D^{h} v\right\|_{t}^{j / h}\left\|v ; L_{p}\left(\Sigma_{R}\right)\right\|^{1-j / h}+C_{5} R^{t}\left\|v ; L_{p}\left(\Sigma_{R}\right)\right\| .
\end{aligned}
$$

Постоянные $C_{4}, C_{5}$ зависят только от размерности пространства.

Отсюда, принимая во внимание (1.6), получим (1.4). Лемма доказана.

Оценки решений сформулированной задачи будут получены на основе известных $L_{p}$-оценок для линейных систем.

Теорема $1.1\left[9\right.$, с. 78]. Пусть $\Omega \in \mathbb{R}^{n}$ - ограниченная область с граничей $\partial \Omega$ класса $C^{t^{\prime}}$. Тогда, если $u_{j} \in W_{p}^{t_{j}}(\Omega) \cap L_{\infty}(\Omega), j=1, \ldots, N,-$ решения задачи (0.7), (0.8) с коэффициентами $a_{\beta}^{i j}(x) \in C^{-s_{i}}(\Omega), 0 \leqslant|\beta| \leqslant s_{i}+t_{j}$, $b_{\tau}^{h j}(x) \in C^{-r_{h}}(\partial \Omega), 0 \leqslant|\tau| \leqslant r_{h}+t_{j}$, то существует постоянная $K>0$, зависящая от $n, N, p, t^{\prime}, \Omega$, постоянной әллиптичности $A$, модулей непрерывности старших коэффициентов в $l_{i j}(x, D)$ и чисел, ограничивающих модули остальных коэффициентов в $l_{i j}(x, D), B_{h j}(x, D)$, такая, что

$$
\begin{aligned}
\sum_{j=1}^{N}\left\|u_{j} ; W_{p}^{t_{j}}(\Omega)\right\| & \leqslant K\left(\sum_{i=1}^{N}\left\|\sum_{k=1}^{N} l_{i k} u_{k} ; W_{p}^{-s_{i}}(\Omega)\right\|\right. \\
& \left.+\sum_{h=1}^{m}\left\|\sum_{k=1}^{N} B_{h k} u_{k} ; W_{p}^{-r_{h}-1 / p}(\partial \Omega)\right\|+\sum_{k=1}^{N}\left\|u_{k} ; L_{p}(\Omega)\right\|\right) .
\end{aligned}
$$




\section{$\S 2 . \quad L_{p}$-оценки решений эллиптической системы квазилинейных уравнений}

В этом параграфе будет найден точный порядок роста нелинейных операторов относительно производных решения, при котором можно установить $L_{p}$-оценки решений. Во всех теоремах этого параграфа предполагаем, что коэффициенты дифференциальных операторов, входящих в $(0.7),(0.8)$, удовлетворяют условиям теоремы 1.1. Докажем сначала внутреннюю оценку.

Tеорема 2.1. Пусть $u=\left(u_{1}, \ldots, u_{N}\right)$ - решение системы $(0.7)$ в ограниченной области $\Omega \subset \mathbb{R}^{n}, u_{j} \in W_{p}^{t_{j}}(\Omega, \mathrm{loc}) \cap L_{\infty}(\Omega),\left\|u_{j} ; L_{\infty}(\Omega)\right\| \leqslant M, j=1, \ldots, N$. Предположим, что

$$
\begin{aligned}
& \sum_{i=1}^{N} \sum_{0 \leqslant|\alpha| \leqslant-s_{i}}\left|D_{x}^{\alpha} f_{i}\left(x, \xi_{0}^{j}(x), \ldots, \xi_{s_{i}+t_{j}-1}^{j}(x)\right)_{j=1, \ldots, N}\right| \\
& \leqslant C\left(x, \xi_{0}^{1}, \ldots, \xi_{0}^{N}\right)\left(1+F\left(\sum_{j=1}^{N} \sum_{k=0}^{t_{j}-1}\left|\xi_{k}^{j}\right|^{t_{j} / k}\right)\right)
\end{aligned}
$$

где $C\left(x, \xi_{0}^{1}, \ldots, \xi_{0}^{N}\right), F(t)$ - ограниченные, измеримье по Борелю функиии, причем

$$
\left|C\left(x, \xi_{0}^{1}, \ldots, \xi_{0}^{N}\right)\right| \leqslant C_{M}
$$

npu $x \in \Omega,\left|\xi_{0}^{j}\right| \leqslant M, u$

$$
F(t)=o(t) \quad n p u \quad t \rightarrow \infty \quad u \quad|F(t)| \leqslant C_{K} \quad n p u \quad|t| \leqslant K .
$$

Тогда для любой строго внутренней подобласти $\Omega^{\prime}$ области $\Omega$ существует функция $\psi$, не зависящая от и, такая, что справедлива оченка

$$
\sum_{j=1}^{N}\left\|u_{j} ; W_{p}^{t_{j}}\left(\Omega^{\prime}\right)\right\| \leqslant \psi(M)
$$

ДоКАЗАТЕЛЬСтво. Если области $\Omega$ и $\Omega^{\prime}$ заданы, то мы можем покрыть $\Omega^{\prime}$ конечным числом шаров, содержашихся в $\Omega$ вместе со своими границами. Поэтому достаточно доказать теорему для случая, когда $\Omega$ и $\Omega^{\prime}$ - концентрические шары малых радиусов $R$ и $r$, соответственно. Не ограничивая общности можно считать, что их центры совпадают с началом координат. В доказательстве $C_{k}, k=1,2, \ldots$, обозначают константы, зависящие от тех же величин, что и постоянная $K$ в теореме 1.1.

Рассмотрим в шаре $\Sigma_{R}$ произвольную функцию $v=\left(v_{1}, \ldots, v_{N}\right)$, имеюшую компактный носитель в шаре $\Sigma_{R-\delta}, \delta>0$, такую, что $v_{j} \in W_{p}^{t^{j}}\left(\Sigma_{R}\right), j=1, \ldots, N$. Применяя к $v$ теорему 1.1 , получим оценку

$$
\sum_{j=1}^{N}\left\|v_{j} ; W_{p}^{t_{j}}\left(\Sigma_{R}\right)\right\| \leqslant C_{0}\left(\sum_{i=1}^{N}\left\|\sum_{k=1}^{N} l_{i k} v_{k} ; W_{p}^{-s_{i}}\left(\Sigma_{R}\right)\right\|+\sum_{k=1}^{N}\left\|v_{k} ; L_{p}\left(\Sigma_{R}\right)\right\|\right) .
$$


Определим в пространстве функций $w \in W_{p}^{k}(\Sigma$, loc $)$

$$
\tilde{\tilde{\|}} D^{k} w\left\|_{t}=\sup _{0<d<R} d^{k+t}\right\| w ; W_{p}^{k}\left(\Sigma_{R-d}\right) \|, \quad t \geqslant 0 .
$$

Заметим, что

$$
\tilde{\|} D^{k} w\left\|_{t} \leqslant \tilde{\tilde{\|}} D^{k} w\right\|_{t}
$$

Допустим, что наибольшее значение величина $\tilde{\tilde{\|}} D^{t_{j}} u_{j} \|_{t^{\prime}-t_{j}}$ принимает для $j=1$. Пусть $d$ такое, что $0<d<R$ и

$$
\tilde{\tilde{\|}} D^{t_{1}} u_{1}\left\|_{t^{\prime}-t_{1}} \leqslant 2 d^{t^{\prime}}\right\| u_{1} ; W_{p}^{k}\left(\Sigma_{R-d}\right) \| .
$$

Пусть функция $\xi(x) \in C_{0}^{\infty}\left(\Sigma_{R}\right),|\xi(x)| \leqslant 1$, причем $\xi \equiv 1$ при $|x|<R-d, \xi \equiv 0$ при $|x|>R-d / 2$, и

$$
\left|D^{k} \xi\right| \leqslant C d^{-k}, \quad 0 \leqslant k \leqslant t^{\prime},
$$

где постоянная $C>0$ зависит только от $k$.

Положим $v_{j}=\xi u_{j}, j=1, \ldots, N$. Очевидно, $v_{j} \in W_{p}^{t_{j}}\left(\Sigma_{R}\right)$ и имеют компактный носитель в $\Sigma_{R-d / 2}$. Поэтому для $v$ справедлива оценка $(2.5)$.

Из условия (2.3) следует, что для любого $\varepsilon>0$ сушествует $K_{\varepsilon}>0$ такое, что $|F(t) / t|<\varepsilon$ при $|t|>K_{\varepsilon}$, и $|F(t)| \leqslant C_{K_{\varepsilon}}$ при $|t| \leqslant K_{\varepsilon}$. Обозначим

$$
F_{\varepsilon}(t)= \begin{cases}0, & |t| \leqslant K_{\varepsilon}, \\ F(t), & |t|>K_{\varepsilon} .\end{cases}
$$

Отсюда, пользуясь условиями $(2.1),(2.2)$ и применяя неравенство Минковского

$$
\left(\int\left(\sum_{i} a_{i}\right)^{p} d x\right)^{1 / p} \leqslant \sum_{i}\left(\int a_{i}^{p} d x\right)^{1 / p}
$$

для $a_{i} \geqslant 0, p>1$, имеем

$$
\begin{aligned}
& \left\|\sum_{k=1}^{N} l_{i k}\left(u_{k} \xi\right) ; W_{p}^{-s_{i}}\left(\Sigma_{R}\right)\right\| \\
& \quad \leqslant C_{M}\left[\left(1+C_{K_{\varepsilon}}\right) \omega_{n} R^{n}+\left\|F_{\varepsilon} ; L_{p}\left(\Sigma_{R}\right)\right\|\right] \\
& \quad \leqslant C_{M}\left[\left(1+C_{K_{\varepsilon}}\right) \omega_{n} R^{n}+\sum_{j=1}^{N} \sum_{k=1}^{t_{j}-1}\left\|D^{k}\left(u_{j} \xi\right) ; L_{p t_{j} / k}\left(\Sigma_{R}\right)\right\|^{t_{j} / k}\right],
\end{aligned}
$$

$\omega_{n}$ - объем единичного шара в $\mathbb{R}^{n}$. Из интерполяционного неравенства (1.1) следует, что

$$
\begin{aligned}
& \sum_{k=1}^{t_{j}-1}\left\|D^{k}\left(u_{j} \xi\right) ; L_{p t_{j} / k}\left(\Sigma_{R}\right)\right\|^{t_{j} / k} \\
& \quad \leqslant \sum_{k=1}^{t_{j}-1}\left(C_{1}\left\|D^{t_{j}}\left(u_{j} \xi\right) ; L_{p}(\Sigma)\right\|\left\|u_{j} \xi ; L_{\infty}\left(\Sigma_{R}\right)\right\|^{t_{j} / k-1}\right. \\
& \left.\quad+C_{2} R^{n / p-t_{j}}\left\|u_{j} \xi ; L_{\infty}\left(\Sigma_{R}\right)\right\|^{t_{j} / k}\right) .
\end{aligned}
$$


Пользуясь интегральным неравенством Минковского, свойствами функции $\xi$ и определением $\tilde{\|} \cdot \|(1.3)$, получим

$$
\begin{aligned}
& \left\|D^{t_{j}}\left(u_{j} \xi\right) ; L_{p}\left(\Sigma_{R}\right)\right\|=\left\|D^{t_{j}}\left(u_{j} \xi\right) ; L_{p}\left(\Sigma_{R-d / 2}\right)\right\| \\
& \quad \leqslant\left\|D^{t_{j}} u_{j} ; L_{p}\left(\Sigma_{R-d / 2}\right)\right\|+C_{3} \sum_{i=1}^{t_{j}}\left\|D^{i} \xi D^{t_{j}-i} u_{j} ; L_{p}\left(\Sigma_{R-d / 2}\right)\right\|^{t_{j} / k} \\
& \quad \leqslant\left\|D^{t_{j}} u_{j} ; L_{p}\left(\Sigma_{R-d / 2}\right)\right\|+C_{4} \sum_{i=1}^{t_{j}} d^{-i}\left\|D^{t_{j}-i} u_{j} ; L_{p}\left(\Sigma_{R-d / 2}\right)\right\| \\
& \quad \leqslant\left(\frac{d}{2}\right)^{-t^{\prime}}\left(\tilde{\|} D^{t_{j}} u_{j}\left\|_{t^{\prime}-t_{j}}+C_{5} \sum_{i=1}^{t_{j}} \tilde{\|} D^{t_{j}-i} u_{j}\right\|\right) .
\end{aligned}
$$

Из неравенства (1.5) с $\varepsilon=1 / 2$ и оценки (2.7) следует, что

$$
\left\|D^{t_{j}}\left(u_{j} \xi\right) ; L_{p}\left(\Sigma_{R}\right)\right\| \leqslant C_{6} d^{-t^{\prime}}\left(\tilde{\|} D^{t_{j}-i} u_{j} \|+M\right) \leqslant C_{6} d^{-t^{\prime}}\left(\tilde{\tilde{\tilde{D}}} D^{t_{1}} u_{1} \|+M\right) .
$$

Таким образом,

$$
\begin{aligned}
& \left\|\sum_{k=1}^{N} l_{i k}\left(u_{k} \xi\right) ; W_{p}^{-s_{i}}\left(\Sigma_{R}\right)\right\| \\
& \leqslant C_{M}\left[\left(1+C_{K_{\varepsilon}}\right) R^{n} \omega_{n}+\varepsilon C_{7} d^{-t^{\prime}}\left(\tilde{\tilde{\|}} D^{t_{1}} u_{1} \|_{t^{\prime}-t_{1}}+M\right) \sum_{j=1}^{N} \sum_{k=1}^{t_{j}-1} M^{t_{j} / k-1}\right. \\
& \left.\quad+\varepsilon C_{2} \sum_{j=1}^{N} \sum_{k=1}^{t_{j}-1} R^{n / p-t_{j}} M^{t_{j} / k}\right] \\
& \leqslant d^{t^{\prime}} C_{8}\left[\omega_{n} R^{n+t^{\prime}}\left(1+C_{K_{\varepsilon}}\right)+\varepsilon \tilde{\tilde{\|}} D^{t_{1}} u_{1} \|_{t^{\prime}-t_{1}} \sum_{j=1}^{N} \sum_{k=1}^{t_{j}-1} M^{t_{j} / k-1}\right. \\
& \left.\quad+\varepsilon \sum_{j=1}^{N} \sum_{k=1}^{t_{j}-1} M^{t_{j} / k}+\varepsilon \sum_{j=1}^{N} \sum_{k=1}^{t_{j}-1} R^{n / p-t_{j}+t^{\prime}} M^{t_{j} / k-1}\right]
\end{aligned}
$$

В силу выбора $d$, используя (2.5), имеем

$$
\begin{aligned}
\tilde{\tilde{\|}} D^{t_{1}} u_{1}\left\|_{t^{\prime}-t_{1}} \leqslant 2 d^{t^{\prime}}\right\| u_{1} ; W_{p}^{t_{1}}\left(\Sigma_{R-d}\right)\left\|\leqslant 2 d^{t^{\prime}}\right\| u_{1} ; W_{p}^{t_{1}}\left(\Sigma_{R}\right) \| \\
\leqslant 2 C_{9}\left[\left(1+C_{K_{\varepsilon}}\right) R^{t^{\prime}+n} \omega_{n}+\varepsilon \tilde{\tilde{\|}} D^{t_{1}} u_{1} \|_{t^{\prime}-t_{1}} \sum_{j=1}^{N} \sum_{k=1}^{t_{j}-1} M^{t_{j} / k-1}\right. \\
\left.+\varepsilon \sum_{j=1}^{N} \sum_{k=1}^{t_{j}-1} M^{t_{j} / k}+\varepsilon \sum_{j=1}^{N} \sum_{k=1}^{t_{j}-1} R^{n / p-t_{j}+t^{\prime}} M^{t_{j} / k-1}+M\right] .
\end{aligned}
$$

Возьмем $\varepsilon=\left(4 C_{9} \sum_{j=1}^{N} \sum_{k=1}^{t_{j}-1} M^{t_{j} / k-1}\right)^{-1}$, тогда

$$
\tilde{\tilde{\|}} D^{t_{1}} u_{1} \|_{t^{\prime}-t_{1}} \leqslant \psi_{0}(M)
$$


Следовательно,

$$
\begin{aligned}
\sum_{j=1}^{N}\left\|u_{j} ; W_{p}^{t_{j}}\left(\Sigma_{r}\right)\right\| & \leqslant(R-r)^{-t^{\prime}} \sum_{j=1}^{N} \tilde{\tilde{\|}} D^{t_{j}} u_{j} \|_{t^{\prime}-t_{j}} \\
& \leqslant N(R-r)^{-t^{\prime}} \tilde{\tilde{\|}} D^{t_{1}} u_{1} \|_{t^{\prime}-t_{1}} \\
& \leqslant N(R-r)^{-t^{\prime}} \psi_{0}(M)=\psi(M) .
\end{aligned}
$$

Теорема доказана.

Следуюший пример покажет, что условие $F(t)=o(t)$ при $t \rightarrow \infty$ существенно, т.е. если $F(t)=O(t)$ при $t \rightarrow \infty$, то оценка (2.4) может не иметь места для ограниченных решений.

Действительно, функции $u_{\lambda}(x)=\sin \lambda x, \lambda \in \mathbb{R}$, в интервале $|x|<1$ удовлетворяют обыкновенному дифференциальному уравнению

$$
u^{(\mathrm{iv})}=u\left[\left(u^{\prime}\right)^{4}+u^{2}\left(u^{\prime \prime}\right)^{2}-2 u\left(u^{\prime}\right)^{2} u^{\prime \prime}\right] .
$$

Правая часть не превосходит по модулю $\left(2|u|+|u|^{2}\right)\left(\left|u^{\prime}\right|^{4}+\left|u^{\prime \prime}\right|^{2}\right)$. Решения $u_{\lambda}$ бесконечно дифференцируемы, и $\left|u_{\lambda}(x)\right| \leqslant 1$. Из теорем вложения Соболева следует, что, если справедлива оценка (2.4), то тогда можно оценить $\left|u_{\lambda}^{\prime}(0)\right|$ некоторой постоянной, не зависяшей от $u_{\lambda}$. Однако $\left|u_{\lambda}^{\prime}(0)\right|=\lambda$ неограниченно растет при $\lambda \rightarrow \infty$.

Аналогично теореме 2.1 доказываются локальные $L_{p}$-оценки решения вблизи гранищы.

Теорема 2.2. Пусть $и=\left(u_{1}, \ldots, u_{N}\right)$ - решение системь (0.7), удовлетворяющее на части $Г$ граничь $\partial \Omega$ области $\Omega$ условиям (0.8):

$$
u_{j} \in W_{p}^{t_{j}}(\Omega, \mathrm{loc}) \cap L_{\infty}(\Omega), \quad\left\|u_{j} ; L_{\infty}(\Omega)\right\| \leqslant M, \quad j=1, \ldots, N .
$$

Предположим, что вьполнено неравенство

$$
\begin{aligned}
\sum_{i=1}^{N} \sum_{0 \leqslant|\alpha| \leqslant-s_{i}}\left|D_{x}^{\alpha} f_{i}\right| & +\sum_{k=1}^{m} \sum_{0 \leqslant|\beta| \leqslant-r_{h}}\left|D_{x}^{\beta} \varphi_{h}\right| \\
& \leqslant C\left(x, \xi_{0}^{1}, \ldots, \xi_{0}^{N}\right)\left(1+F\left(\sum_{j=1}^{N} \sum_{k=0}^{t_{j}-1}\left|\xi_{k}^{j}\right|^{t_{j} / k}\right)\right)
\end{aligned}
$$

где функиии $C\left(x, \xi_{0}^{1}, \ldots, \xi_{0}^{N}\right), F(t)$ такие же, как и в теореме 2.1. Тогда для любой подобласти $\Omega^{\prime}$ области $\Omega$ такой, что $\overline{\partial \Omega^{\prime} \cap \partial \Omega} \subset \Gamma$ справедливо неравенство

$$
\sum_{j=1}^{N}\left\|u_{j} ; W_{p}^{t_{j}}\left(\Omega^{\prime}\right)\right\| \leqslant \psi_{1}(M),
$$

где $\psi_{1}$ - функция, не зависящая от и. 
ДокАЗАТЕЛЬСтво. Так как доказательство теоремы проводится по той же схеме, что и доказательство теоремы 2.1 , то наметим лиш основные его этапы.

Заметим, что теорему достаточно доказать для случая, когда $\Omega=\Sigma_{R}$-полушар радиуса $R, \Gamma=\mathscr{F}_{R}$, а $\Omega^{\prime}=\Sigma_{r}$, где $r<R$. Переход к произвольной области производится стандартным методом [8], [9].

Рассмотрим функции $v_{j} \in W_{p}^{t_{j}}\left(\Sigma_{R}\right)$, обрашающиеся в нуль вне $\Sigma_{R-\varepsilon}, \varepsilon>0$. Применим к $v=\left(v_{1}, \ldots, v_{N}\right)$ теорему 1.1:

$$
\begin{aligned}
\sum_{j=1}^{N}\left\|v_{j} ; W_{p}^{t_{j}}\left(\Sigma_{R}\right)\right\| & \leqslant C_{1}\left(\sum_{i=1}^{N}\left\|\sum_{k=1}^{N} l_{i k} v_{k} ; W_{p}^{-s_{i}}\left(\Sigma_{R}\right)\right\|\right. \\
+ & \left.\sum_{h=1}^{m}\left\|\sum_{k=1}^{N} B_{h k} v_{k} ; W_{p}^{-r_{h}-1 / p}\left(\mathscr{F}_{R}\right)\right\|+\sum_{k=1}^{N}\left\|v_{k} ; L_{p}\left(\Sigma_{R}\right)\right\|\right) .
\end{aligned}
$$

Определим в пространстве функций $w \in W_{p}^{t_{j}}\left(\Sigma_{R} \cup \mathscr{F}_{R}\right.$, loc $)$

$$
\tilde{\tilde{\|}} D^{k} w\left\|_{t}=\sup _{0<d<R} d^{k+t}\right\| w ; W_{p}^{k}\left(\Sigma_{R-d}\right) \|, \quad t \geqslant 0 .
$$

Заметим, что

$$
\tilde{\|} D^{k} w\left\|_{t} \leqslant \tilde{\tilde{\|}} D^{k} w\right\|_{t} .
$$

Допустим, что наибольшее значение величина $\tilde{\tilde{\|}} D^{t_{j}} u_{j} \|_{t^{\prime}-t_{j}}$ принимает для $j=1$. Пусть $d$ такое, что $0<d<R$ и

$$
\tilde{\tilde{\|}} D^{t_{1}} u_{1}\left\|_{t^{\prime}-t_{1}} \leqslant 2 d^{t^{\prime}}\right\| u_{1} ; W_{p}^{k}\left(\Sigma_{R-d}\right) \| .
$$

Положим $v_{j}=\xi u_{j}, j=1, \ldots, N$, где $\xi$ - функция, определенная в теореме 2.1 . Очевидно, для $v=\left(v_{1}, \ldots, v_{N}\right)$ выполнена оценка (2.10). Заметим, что

$$
\begin{aligned}
\left\|\sum_{k=1}^{N} B_{h k} v_{k} ; W_{p}^{-r_{h}-1 / p}\left(\mathscr{F}_{R}\right)\right\| & =\left\|\sum_{k=1}^{N} B_{h k}\left(u_{k} \xi\right) ; W_{p}^{-r_{h}-1 / p}\left(\mathscr{F}_{R}\right)\right\| \\
& \leqslant\left\|\sum_{k=1}^{N} B_{h k}\left(u_{k} \xi\right) ; W_{p}^{-r_{h}}\left(\Sigma_{R}\right)\right\| .
\end{aligned}
$$

Далее рассуждаем так же, как при доказательстве теоремы 2.1.

Непосредственно из теоремы 1.1 и интерполяционных неравенств Гальярдо-Ниренберга следует справедливость глобальной оценки решения вплоть до границы. А именно, имеет место следуюшая теорема.

Теорема 2.3. Пусть функиии $u_{j} \in W_{p}^{t_{j}}(\Omega) \cap L_{\infty}(\Omega), j=1, \ldots, N$, удовлетворяют системе (0.7) в области $\Omega \in \mathbb{R}^{n}$ и краевым условиям (0.8) на граниче $\partial \Omega$ класса $C^{t^{\prime}}$. Предполагаем, что $\left\|u_{j} ; L_{\infty}(\Omega)\right\| \leqslant M$ и выполнено условие $(2.9)$ с функииями $C\left(x, \xi_{0}, \ldots, \xi_{N}\right)$ и $F(t)$, определенными в теореме 2.1. Тогда существует функиия $\psi_{2}$, не зависящая от и, такая, что справедливо неравенство

$$
\sum_{j=1}^{N}\left\|u_{j} ; W_{p}^{t_{j}}(\Omega)\right\| \leqslant \psi_{2}(M) .
$$




\section{§3. О гладкости решений внутри области}

При некоторых ограничениях на гладкость коэффициентов в уравнениях на соотношения между числами $t_{j}, p, n$ в зависимости от характера роста $f_{i}$ относительно производных решения получим результаты о повышении гладкости решений внутри области. В ходе доказательств теорем этого параграффа будет использоваться следуюшая лемма об интегралах типа потенциала.

ЛЕмма 3.1 [13]. Пусть функиия $\varphi$ от $n$ переменных с носителем в шаре $\Sigma_{1}$ принадлежст $L_{p}, 1<p<\infty ;$ рассмотрим функиию

$$
g(x)=\int \frac{\varphi(y)}{|x-y|^{\lambda}} d y, \quad \lambda<n
$$

положим $a=n-\lambda-n / p$.

Тогда:

1. если $a<0,1 / q=-a / n$, mo

$$
\left\|g ; L_{q}\left(\Sigma_{1}\right)\right\| \leqslant \text { Const }\left\|\varphi ; L_{p}\left(\Sigma_{1}\right)\right\| ;
$$

2. если $a=0$, то

$$
\left\|g ; L_{q}\left(\Sigma_{1}\right)\right\| \leqslant \text { Const }\left\|\varphi ; L_{p}\left(\Sigma_{1}\right)\right\|
$$

при любом конечном $q>0$;

3. если $a>0$ иа - не челое число, то

$$
\left\|g ; C^{[a], a-[a]}\left(\Sigma_{1}\right)\right\| \leqslant \text { Const }\left\|\varphi ; L_{p}\left(\Sigma_{1}\right)\right\| ;
$$

4. если $a>0-$ челое число, то

$$
\left\|g ; C^{a-1, b}\left(\Sigma_{1}\right)\right\| \leqslant \text { Const }\left\|\varphi ; L_{p}\left(\Sigma_{1}\right)\right\|
$$

для любого $0 \leqslant b<1$.

Здесь постоянные зависят только от $n, \lambda, p, q, b$.

ТЕОРема 3.1. Пусть функции $u_{1}, \ldots, u_{N}$ удовлетворяют в области $\Omega \subset \mathbb{R}^{n}$ системе (0.1) с коэффициентами $a_{\beta}^{i j} \in C^{-s_{i}, \sigma}(\Omega),|\beta|=0, \ldots, s_{i}+t_{j}, 1 \leqslant i \leqslant N$,

$1 \leqslant j \leqslant N$, где $\sigma \in(0,1)$. Предположим, ито $u_{j} \in W_{p}^{t_{j}}(\Omega, \operatorname{loc}) \cap L_{\infty}(\Omega)$, $\left\|u_{j} ; L_{\infty}(\Omega)\right\| \leqslant M, j=1, \ldots, N$, и выполнено неравенство

$$
\begin{aligned}
& \sum_{i=1}^{N} \sum_{0 \leqslant|\alpha| \leqslant-s_{i}}\left|D_{x}^{\alpha} f_{i}\left(x, \xi_{0}^{j}(x), \ldots, \xi_{N}^{j}(x)\right)\right| \\
& \leqslant C\left(x, \xi_{0}^{j}, \ldots, \xi_{0}^{N}\right)\left(1+\sum_{j=1}^{N} \sum_{k=0}^{k_{j}-1}\left|\xi_{k}^{j}\right|^{t_{j} / k(1-\varepsilon)}\right),
\end{aligned}
$$

əде $0<\varepsilon<1, C\left(x, \xi_{0}^{1}, \ldots, \xi_{0}^{N}\right)$ - ограниченная, измеримая по Борелю функиия, такая, что

$$
\left|C\left(x, \xi_{0}^{1}, \ldots, \xi_{0}^{N}\right)\right| \leqslant C_{M}
$$


при $x \in \Omega,\left|\xi_{0}^{j}\right| \leqslant M$.

Тогда в каждой строго внутренней подобласти $\Omega^{\prime}$ области $\Omega\left(\Omega^{\prime} \subset\right.$ $\left.\overline{\Omega^{\prime}} \subset \Omega\right), u_{j} \in C^{t_{j}-1, \delta}\left(\Omega^{\prime}\right)$, әде $\delta$ - любое число из интервала $(0,1)$, причем справедлива оченка

$$
\sum_{j=1}^{N}\left\|u_{j} ; C^{t_{j}-1, \delta}\left(\Omega^{\prime}\right)\right\| \leqslant \psi(M),
$$

әде функция $\psi$ не зависит от и.

ДокАЗАТЕЛЬСтво. Так как все рассмотрения локальные, то можно считать, что область $\Omega$ содержится в единичном шаре $\Sigma_{1}$. Не ограничивая обшности предположим, что операторы $l_{i j}(x, D)$ в системе $(0.1)$ содержат только члены порядка $s_{i}+t_{j}$. Пусть $L(x, D)$ - линейный дифференциальный оператор порядка $2 m$, содержаший только старшие члены, соответствуюший многочлену

$$
L(x, \xi)=\operatorname{det}\left(l_{i j}(x, \xi)\right) .
$$

Для любой фиксированной точки $x_{0} \in \Omega$ пусть $\Gamma\left(x_{0}, x-y\right)$ - фундаментальное решение оператора $L(x, D)$, коэффициенты которого взяты в точке $x_{0}$. Известно [14], что справедливы следуюшие неравенства:

$$
\left|D_{x}^{s} \Gamma(x-y)\right| \leqslant \text { Const }|x-y|^{2 m-n-s}
$$

в случаях: 1) $s \geqslant 0$ и $n$ либо четно и больше $2 m$, либо нечетно; 2) $s>2 m-n$, $n \leqslant 2 m$ и четно. Если $n \leqslant 2 m$ четно и $0 \leqslant s \leqslant 2 m-n$, то

$$
\left|D_{x}^{s} \Gamma(x-y)\right| \leqslant \text { Const }|x-y|^{2 m-n-s}(1+\log |x-y|) .
$$

Константы в $(3.3),(3.4)$ зависят только от $s, m, n$, постоянной эллиптичности $A$.

Для функций $v_{j}, j=1, \ldots, N$, с компактными носителями в $\Omega$, имеющих обобщенные производные до порядка $t_{j}$ включительно, интегрируемые со степенью $p>1$, справедливо представление [9]

$$
\begin{aligned}
v_{j}= & \int_{\Omega} \Gamma\left(x_{0}, x-y\right) \sum_{i=1}^{N} L^{j i}\left(x_{0}\right) \sum_{k=1}^{N} l_{i k}\left(x_{0}\right) v_{k}(y) d y \\
= & \int_{\Omega} \Gamma\left(x_{0}, x-y\right) \sum_{i=1}^{N} L^{j i}\left(x_{0}\right) \sum_{k=1}^{N}\left(l_{i k}\left(x_{0}\right)-l_{i k}(y)\right) v_{k}(y) d y \\
& +\int_{\Omega} \Gamma\left(x_{0}, x-y\right) \sum_{i=1}^{N} L^{j i}\left(x_{0}\right) \sum_{k=1}^{N} l_{i k}(y) v_{k}(y) d y .
\end{aligned}
$$

Здесь $L^{j i}(x)=L^{j i}(x, D)$ - оператор порядка $2 m-s_{i}-t_{j}$ такой, что

$$
\sum_{j=1}^{N} l_{k j} L^{j i}=\delta_{i}^{k} L
$$

где $\delta_{i}^{k}$ - символ Кронекера. 
Для каждой внутренней подобласти $\Omega^{\prime}$ области $\Omega$ определим неотрицательную функцию $\xi$ с компактным носителем в $\Omega$, бесконечно гладкую и тождественно равную 1 в $\Omega^{\prime}$. Положим $v_{j}=\xi u_{j}, j=1, \ldots, N$. Тогда

$$
\begin{aligned}
& D^{s}\left(\xi(x) u_{j}(x)\right)=\int_{\Omega} D_{x}^{2 m-t_{j}+s} \Gamma\left(x_{0}, x-y\right) \sum_{i=1}^{N} b_{i}\left(x_{0}\right) D^{-s_{i}} \sum_{k=1}^{N} l_{i k}(y)\left(\xi(y) u_{k}(y)\right) d y \\
& \quad+\int_{\Omega} D_{x}^{2 m-t_{j}+s} \Gamma\left(x_{0}, x-y\right) \sum_{i=1}^{N} b_{i}\left(x_{0}\right) D^{-s_{i}} \sum_{k=1}^{N}\left(l_{i k}\left(x_{0}\right)-l_{i k}(y)\right)\left(\xi(y) u_{k}(y)\right) d y \\
& \quad+C_{s j}\left(x_{0}\right) \sum_{i=1}^{N} b_{i}\left(x_{0}\right) D^{-s_{i}} \sum_{k=1}^{N} l_{i k}\left(x_{0}\right)\left(\xi(x) u_{k}(x)\right),
\end{aligned}
$$

где $b_{i}$ обозначают известные функции класса $C^{0, \sigma}\left(\Omega^{\prime}\right), C_{s j}$ - постоянная, равная нулю при $s<t_{j}, j=1, \ldots, N$, и при $s=t_{j}$ интегралы берутся в смысле главного значения по Коши. Положим теперь $x_{0}=x \in \Omega^{\prime}$, тогда при $0 \leqslant s \leqslant t_{j}$

$$
\begin{aligned}
& D^{s} u_{j}(x)=\int_{\Omega} D_{x}^{2 m-t_{j}+s} \Gamma(x, x-y) \sum_{i=1}^{N} b_{i}(x) D^{-s_{i}} \sum_{k=1}^{N} l_{i k}(y)\left(\xi(y) u_{k}(y)\right) d y \\
& +\int_{\Omega} D_{x}^{2 m-t_{j}+s} \Gamma(x, x-y) \sum_{i=1}^{N} b_{i}(x) D^{-s_{i}} \sum_{k=1}^{N}\left(l_{i k}(x)-l_{i k}(y)\right)\left(\xi(y) u_{k}(y)\right) d y \\
& \quad+C_{s j}(x) \sum_{i=1}^{N} b_{i}(x) D^{-s_{i}} \sum_{k=1}^{N} l_{i k}(x) u_{k}(x)
\end{aligned}
$$

где в выражения $D_{x}^{2 m-t_{j}+s} \Gamma(x, x-y)$ входят дифференцирования только по второму аргументу. Запишем последнее равенство в виде:

$$
D^{s} u_{j}(x)=J_{1, s}^{j}(x)+J_{2, s}^{j}(x)+J_{3, s}^{j}(x),
$$

где

$$
\begin{aligned}
J_{1, s}^{j}(x)= & \int_{\Omega} D_{x}^{2 m-t_{j}+s} \Gamma(x, x-y) \\
& \times \sum_{i=1}^{N} b_{i}(x) \xi(y) D^{-s_{i}} f_{i}\left(y, D^{\gamma} u_{j}(y)\right) d y \\
& \quad+C_{s j}(x) \sum_{i=1}^{N} b_{i}(x) D^{-s_{i}} f_{i}\left(x, D^{\gamma} u_{j}(x)\right), \\
J_{2, s}^{j}(x)= & \int_{\Omega} D_{x}^{2 m-t_{j}+s} \Gamma(x, x-y) \\
& \times \sum_{i, k=1}^{N} b_{i}(x) \sum_{|\beta|+|\gamma| \leqslant t_{k}} C_{\beta \gamma}(y) D^{\gamma} u_{k}(y) D^{\beta} \xi(y) d y \\
|\gamma|<t_{k} & \int_{\Omega} D_{x}^{2 m-t_{j}+s} \Gamma(x, x-y) \\
& \times \sum_{i, k=1}^{N} b_{i}(x) \sum_{|\beta|=t_{k}}\left(a_{\beta}^{i k}(x)-a_{\beta}^{i k}(y)\right) D^{\beta}\left(\xi(y) u_{k}(y)\right) d y,
\end{aligned}
$$


$a_{\beta}^{i k}$ обозначают коэффициенты в операторах $l_{i k}(x, D)$. Так как $a_{\beta}^{i k} \in C^{-s_{i}, \sigma}$ и $-s_{i} \geqslant 0$, то

$$
\left|a_{\beta}^{i k}(x)-a_{\beta}^{i k}(y)\right| \leqslant \text { Const }|x-y|^{\sigma}, \quad 1 \leqslant i \leqslant N, \quad 1 \leqslant k \leqslant N .
$$

Учитывая (3.3), (3.4), имеем оценку

$$
\left|J_{3, s}^{j}(x)\right| \leqslant \text { Const } \int_{\Omega} \frac{1}{|x-y|^{n-\sigma}} \sum_{k=1}^{N} \sum_{|\beta|=t_{k}}\left|D^{\beta}\left(\xi(y) u_{k}(y)\right)\right| d y .
$$

Непосредственно из теорем вложения Соболева следует:

1) если $p \geqslant n$, то $u_{j} \in W_{q}^{t_{j}-1}(\Omega$, loc) для любого $q>1$, и выполнены неравенства

$$
\left\|u_{j} ; W_{q}^{t_{j}-1}\left(\Omega^{\prime}\right)\right\| \leqslant \text { Const }\left\|u_{j} ; W_{p}^{t_{j}-1}\left(\Omega^{\prime \prime}\right)\right\|
$$

для любой $\Omega^{\prime} \subset \overline{\Omega^{\prime}} \subset \Omega^{\prime \prime} \subset \Omega$;

2) если $p<n$, то $u_{j} \in W_{p_{0}}^{t_{j}-1}(\Omega$, loc $)$, где $p_{0}=(1 / p-1 / n)^{-1}$, и выполнены неравенства

$$
\left\|u_{j} ; W_{p_{0}}^{t_{j}-1}\left(\Omega^{\prime}\right)\right\| \leqslant \text { Const }\left\|u_{j} ; W_{p}^{t_{j}-1}\left(\Omega^{\prime \prime}\right)\right\|
$$

для любой $\Omega^{\prime} \subset \overline{\Omega^{\prime}} \subset \Omega^{\prime \prime} \subset \Omega$.

Заметим, что постоянные в этих неравенствах зависят от области $\Omega^{\prime \prime}$, но не зависят от функций $u_{j}$.

Рассмотрим производные $D^{t_{j}} u_{j}, j=1, \ldots, N$, в случае $p=n$. Учитывая условие (3.1) и оценки (3.3), (3.4), мы найдем по теореме Зигмунда-Кальдерона [15], что $J_{1, t_{j}}^{j} \in L_{q}(\Omega$, loc $), J_{2, t_{j}}^{j} \in L_{q}(\Omega$, loc $)$ для любого конечного $q>0$. Принимая во внимание (3.5), по лемме 3.1 имеем, что $J_{3, t_{j}}^{j} \in L_{q_{1}}\left(\Omega\right.$, loc) при $1 / q_{1}=1 / p-\sigma / n$.

Таким образом, $u_{j} \in W_{q_{1}}^{t_{j}}(\Omega, \mathrm{loc}), q_{1}>p=n$. Далее получим, что $u_{j} \in$ $W_{q_{2}}^{t_{j}}(\Omega, \mathrm{loc})$, где

$$
\frac{1}{q_{2}}=\frac{1}{q_{1}}-\frac{\sigma}{n}=\frac{1}{p}-\frac{2 \sigma}{n}
$$

и т. д., $u_{j} \in W_{q_{k}}^{t_{j}}(\Omega, \mathrm{loc})$, где $1 / q_{k}=-a_{k} / n=1 / p-k \sigma / n$, и, следовательно, через конечное число шагов придем к $a_{k} \geqslant 0$. Тогда из леммы 3.1 будем иметь, что $u_{j} \in W_{q}^{t_{j}}(\Omega)$ для любого конечного $q>0$, откуда, применяя теорему вложения Соболева и затем теорему 2.1 , получим, что $u_{j} \in C^{t_{j}-1, \delta}(\Omega$, loc $)$ для любого $\delta \in(0,1)$ и выполнена оценка (3.2).

Очевидно, доказанное утверждение имеет место и для $p>n$.

Вернемся к случаю $p<n$. Докажем, что $u_{j} \in W_{p_{1}}^{t_{j}}(\Omega, \mathrm{loc})$ с некоторым $p_{1}>p$. Из теорем вложения следует, что $u_{j} \in W_{p_{0}}^{t_{j}-1}(\Omega$, loc). Пользуясь оценками $(3.3),(3.4)$, получим, что $J_{3, t_{j}}^{j} \in L_{p_{0}^{\prime}}(\Omega$, loc $), p_{0}^{\prime}=(1 / p-\sigma / n)^{-1}$ по лемме 3.1 , а $J_{2, t_{j}}^{j} \in L_{p_{0}}(\Omega$, loc) по теореме Зигмунда-Кальдерона. Из условия (3.1) и интерполяционного неравенства (1.1) мы найдем согласно теореме Зигмунда-Кальдерона, 
что $J_{1, t_{j}}^{j} \in L_{q_{0}}(\Omega$, loc $)$, где $q_{0}=p /(1-\varepsilon)$. Итак, $u_{j} \in W_{p_{1}}^{t_{j}}(\Omega$, loc $), p_{1}>p$, где $p_{1}=\min \left(q_{0}, p_{0}, p_{0}^{\prime}\right)$, причем

$$
\left\|u_{j} ; W_{p_{1}}^{t_{j}}\left(\Omega^{\prime}\right)\right\| \leqslant \text { Const } \sum_{k}\left\|u_{k} ; W_{p}^{t_{k}}\left(\Omega^{\prime \prime}\right)\right\| .
$$

Если $p_{1}>n$, то все доказано. В противном случае придем к этому за конечное число шагов. Теорема доказана.

ЗАМЕчАНИЕ 3.1. Одновременно мы доказали, что $u_{j} \in W_{q}^{t_{j}}(\Omega$, loc) для любого конечного $q>1$.

ТЕОРемА 3.2. Пусть функиии $u_{1}, \ldots, u_{N}$ удовлетворяют в ограниченной области $\Omega \subset \mathbb{R}^{n}$ системе (0.7) с коэффиииентами $a_{\beta}^{i j} \in C^{-s_{i}, \sigma}(\Omega),|\beta|=$ $0, \ldots, s_{i}+t_{j}, i, j=1, \ldots, N$, где $\sigma \in(0,1)$. Предполохсим, что $u_{j} \in W_{p}^{t_{j}}(\Omega$, lос $) \cap$ $L_{\infty}(\Omega),\left\|u_{j} ; L_{\infty}(\Omega)\right\| \leqslant M, t_{j} p>n, j=1, \ldots, N$. Предположсим, что выполнены условия (2.1), (2.2), (2.3). Тогда $u_{j} \in C^{t_{j}-1, \delta}(\Omega, \operatorname{loc})$, и для любой внутренней подобласти $\Omega^{\prime}\left(\Omega^{\prime} \subset \overline{\Omega^{\prime}} \subset \Omega\right)$ справедлива оченка

$$
\sum_{j=1}^{N}\left\|u_{j} ; C^{t_{j}-1, \delta}\left(\Omega^{\prime}\right)\right\| \leqslant \psi_{4}(M)
$$

әде функиия $\psi_{4}$ не зависит от $u, \delta$ - любое число из интервала $(0,1)$.

ДокАЗАтЕльство. Отличие от доказательства предыдушей теоремы состоит только в несколько ином применении интерполяционного неравенства Гальярдо-Ниренберга (1.1).

Итак, пусть доказано, что $u_{j} \in W_{p_{0}}^{t_{j}-1}(\Omega, l o c), j=1, \ldots, N$. Воспользуемся условиями (2.1), (2.2), (2.3). Легко видеть, что для доказательства теоремы достаточно показать принадлежность функций $\left|D^{k} u_{j}\right|^{t_{j} / k}$ пространству $L_{q_{j}}(\Omega$, loc) с $q_{j}>p$ при $k=1, \ldots, t_{j}-1$. Из леммы 1.1 следует, что для $0<k<t_{j}-1$

$$
\left\|D^{k} u_{j} ; L_{q_{j} t_{j} / k}\left(\Omega^{\prime}\right)\right\| \leqslant C_{1}\left\|D^{t_{j}-1} u_{j} ; L_{p_{0}}\left(\Omega^{\prime}\right)\right\|^{k /\left(t_{j}-1\right)} M^{1-k /\left(t_{j}-1\right)}+C_{2} M
$$

где

$$
q_{j}=\frac{t_{j}-1}{t_{j}} p_{0}
$$

Получаем, что $u_{j} \in W_{q_{j}}^{t_{j}}(\Omega, \mathrm{loc})$ с показателем $q_{j}>p$, поскольку $t_{j} p>n$. Итак, $u_{j} \in W_{p_{1}}^{t_{j}}(\Omega$, loc $)$, где

$$
p_{1}=\min _{j} \frac{t_{j}-1}{t_{j}} p_{0} .
$$

ЗАМЕЧАНИЕ 3.2. Если в условии теоремы потребовать, чтобы выполнялось условие $F(t)=O(t)$ при $t \rightarrow \infty$, то можно утверждать, что $u_{j} \in C^{t_{j}-1, \delta}\left(\Omega^{\prime}\right)$. Однако, как показывает пример, приведенный в $\S 2$, нельзя оценить $\left\|u_{j} ; C^{t_{j}-1, \delta}\left(\Omega^{\prime}\right)\right\|$ через известные величины. 


\section{§4. Теорема об устранимой особенности}

Пусть $\Omega \subset \mathbb{R}^{n}$ - ограниченная область, точка $\mathscr{O} \in \Omega$. В дальнейшем, не ограничивая обшности, будем считать, что точка $\mathscr{O}$ совпадает с началом координат. Будем использовать функциональное пространство $\stackrel{\circ}{H}_{a}^{k}(\Omega)$ - пополнение пространства $C_{0}^{\infty}(\Omega)$ по норме

$$
\left\|u ; \stackrel{\circ}{H}_{a}^{k}(\Omega)\right\|=\left(\sum_{|\alpha| \leqslant k} \int_{\Omega} r^{2(a+|\alpha|-k)}\left|D^{\alpha} u\right|^{2} d x\right)^{1 / 2},
$$

где $r$ - расстояние от $x=\left(x_{1}, \ldots, x_{n}\right)$ до точки $\mathscr{O}$.

В области $\Omega \backslash\{\mathscr{O}\}$ рассмотрим эллиптическую систему дифференциальных уравнений

$$
\sum_{j=1}^{N} l_{h j}(x, D) u_{j}(x)=g_{h}(x), \quad h=1, \ldots, N,
$$

с коэффициентами $a_{\beta}^{h j}(x) \in C^{0, \sigma}(\Omega), \sigma \in(0,1)$. Не ограничивая общности, можно считать, что операторы $l_{h j}$ порядка $t_{j}$ содержат только старшие члены, $t_{j}>0$, $\max _{j} t_{j}=t^{\prime}, \sum_{j=1}^{N} t_{j}=2 m$.

Будем также рассматривать систему с постоянными коэффициентами

$$
\sum_{j=1}^{N} l_{h j}(0, D) u_{j}(x)=g_{h}(x), \quad h=1, \ldots, N
$$

Пусть $\left(r, \omega_{1}, \ldots, \omega_{n-1}\right)$ - полярные координаты, где $r=|x|=\left(\sum_{i=1}^{n} x_{i}^{2}\right)^{1 / 2}$, $\omega_{i}=\omega_{i}\left(y_{1}, \ldots, y_{n}\right), i=1, \ldots, n-1, y_{i}=x_{i} / r, 0<r<\infty, \omega_{i} \in C^{\infty}\left(S_{1}^{n-1}\right), S_{1}^{n-1}$ - $(n-1)$-мерная сфера радиуса 1.

В полярных координатах система (4.2) запишется в форме

$$
\sum_{j=1}^{N} r^{-t_{j}} \mathscr{L}_{h j}\left(\omega, r D_{r}, D_{\omega}\right) u_{j}(r, \omega)=g_{h}(r, \omega), \quad h=1, \ldots, N .
$$

Введем оператор с комплексным параметром $\lambda$ на сффере $S_{1}^{n-1}$

$$
\mathscr{L}(\lambda)=\left(\mathscr{L}_{h j}\left(\omega, i\left(\lambda-i t_{j}\right), D_{\omega}\right)\right)_{h, j=1}^{N} .
$$

Оператор $\mathscr{L}(\lambda)$ действует из пространства $\prod_{j=1}^{N} W_{2}^{t_{j}}\left(S_{1}^{n-1}\right)$ в пространство $\prod_{h=1}^{N} L_{2}\left(S_{1}^{n-1}\right)$. Рассмотрим обратный к нему оператор $\mathscr{L}^{-1}(\lambda)$, действующий из $\prod_{h=1}^{N} L_{2}\left(S_{1}^{n-1}\right)$ в $\prod_{j=1}^{N} W_{2}^{t_{j}}\left(S_{1}^{n-1}\right)$ и являющийся конечно-мероморфной функцией $\lambda[16]$.

Будем использовать известную теорему об асимптотике решения системы (4.1) [17], [18] в следуюшей форме.

ТЕОРема 4.1. Пусть $u=\left(u_{1}, \ldots, u_{N}\right)$ - решение системъ (4.1) в $\Omega \backslash\{\mathscr{O}\}$, причем $u \in \stackrel{\circ}{H} \vec{a}(\Omega)=\prod_{j=1}^{N} \stackrel{\circ}{H}_{a}^{t}(\Omega), g \in \stackrel{\circ}{H}_{a_{1}}^{0}(\Omega)=\prod_{h=1}^{N} \stackrel{\circ}{H}_{a_{1}}^{0}(\Omega), \sigma>a-a_{1}>0$, 
u пусть прямие $\operatorname{Im} \lambda=a+n / 2=b u \operatorname{Im} \lambda=a_{1}+n / 2=b_{1}$ не содержат полюсов оператор-функиии $\mathscr{L}^{-1}(\lambda)$. Тогда

$$
u_{j}(r, \omega)=\sum_{\mu=1}^{K} \sum_{q=0}^{p_{\mu}-1} r^{t_{j}+i \lambda_{\mu}(\log r)^{q}} \psi_{\mu, q, j}(\omega)+w_{j}(r, \omega), \quad 1 \leqslant j \leqslant N,
$$

где $\lambda_{\mu}, \mu=1, \ldots, K,-$ собственнье числа оператора $\mathscr{L}(\lambda)$, лежащие в полосе

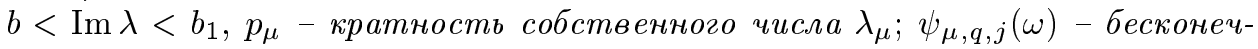
но-дифференцируемые функци ; $w(r, \omega)=\left(w_{1}, \ldots, w_{N}\right)$ - функция из пространства $\stackrel{\circ}{H}_{a_{1}}^{\vec{t}}(\Omega)$. Вектор-функция

$$
v(r, \omega)=\sum_{\mu=1}^{K} \sum_{q=0}^{p_{\mu}-1} r^{\vec{t}+i \lambda_{\mu}}(\log r)^{q} \psi_{\mu, q, j}(\omega)
$$

является решением однородной системь (4.2) в $\Omega \backslash\{\mathscr{O}\}$.

Используя явный вид фундаментального решения для эллиптического оператора, покажем, что полюсы функции $\mathscr{L}^{-1}(\lambda)$ располагаются на мнимой оси $\operatorname{Re} \lambda=0$ в целочисленных точках.

Как и ранее, обозначим

$$
L(0, \xi)=\operatorname{det}\left(l_{h j}(0, \xi)\right) .
$$

Пусть $\Gamma(x)$ - фундаментальное решение оператора $L(0, D)$. Тогда

$$
\sum_{j=1}^{N} l_{h j} L_{h j} \Gamma(x)=\delta(x),
$$

где $L_{h j}$ - алгебраическое дополнение к $l_{h j}$ в матрице $\left(l_{h j}\right)_{h, j=1}^{N}$. Пусть $\lambda_{0}-$ полюс оператора $\mathscr{L}^{-1}(\lambda)$ кратности $p_{0}$. Так как $v(r, \omega)$ - решение однородной системы (4.2) в $\Omega \backslash\{\mathscr{O}\}$, то пользуясь теоремой о представлении функционала, сосредоточенного в точке, получим, принимая во внимание (4.3),

$$
\begin{aligned}
\sum_{j=1}^{N} l_{h j} \sum_{q=0}^{p_{0}-1} r^{t_{j}+i \lambda_{0}}(\log r)^{q} \psi_{0, q, j}(\omega) & =\sum_{|\beta| \leqslant k} C_{\beta} D^{\beta} \delta(x) \\
& =\sum_{j=1}^{N} l_{h j}\left[\sum_{|\beta| \leqslant k} C_{\beta} D^{\beta}\left(L_{h j} \Gamma(x)\right)+\tilde{w}_{j}(x)\right],
\end{aligned}
$$

где $\tilde{w}(x)=\left(\tilde{w}_{1}(x), \ldots, \tilde{w}_{N}(x)\right)$ - гладкое решение. Следовательно,

$$
\sum_{q=0}^{p_{0}-1} r^{t_{j}+i \lambda_{0}}(\log r)^{q} \psi_{0, q, j}(\omega)=\sum_{|\beta| \leqslant k} C_{\beta} D^{\beta}\left(L_{h j} \Gamma(x)\right)+\sum_{\gamma \geqslant 0} d_{\gamma} r^{\gamma} .
$$

Фундаментальное решение $\Gamma(x)$ представимо в виде [14]

$$
\Gamma(x)=\varphi(\omega)\left(r^{2 m-n}+Q(r) \log r\right),
$$

где $Q(r)$ - многочлен степени $2 m-n$, если $2 m \geqslant n$ и $n$ - четно, и $Q \equiv 0$, если $2 m<n$ или $n-$ нечетно, а функция $\psi(\omega) \in C^{\infty}\left(S_{1}^{n-1}\right)$. Отсюда и из (4.4) получим, что $\lambda_{0}$ может находиться только на мнимой оси $\operatorname{Re} \lambda=0$ в точке с целочисленной координатой и иметь кратность 1 или 2. 
ТЕОРема 4.2. Пусть вектор-функиия $u=\left(u_{1}, \ldots, u_{N}\right)$ с компонентами $u_{j} \in W_{p}^{t_{j}}(\Omega \backslash\{\mathscr{O}\}, \mathrm{loc}) \cap L_{\infty}(\Omega)$ - решение эллиптической системы уравнений

$$
\sum_{j=1}^{N} l_{h j}(x, D) u_{j}(x)=f_{h}\left(x, D^{\gamma} u_{j}(x)\right)_{|\gamma| \leqslant s_{h}+t_{j}-1, j=1, \ldots, N}, \quad h=1, \ldots, N
$$

в области $\Omega \backslash\{\mathscr{O}\}$. Предположим, что операторы $l_{h j}$ порядка $t_{j}$ с коэффициентами из $C^{0, \sigma}(\Omega), \sigma \in(0,1)$, содержат только стариие членьи, и выполнено условие

$$
\begin{aligned}
& \sum_{h=1}^{N}\left|f_{h}\left(x, \xi_{0}^{j}, \ldots, \xi_{t_{j}-1}^{j}\right)_{j=1, \ldots, N}\right| \\
& \quad \leqslant C\left(x, \xi_{0}^{1}, \ldots, \xi_{0}^{N}\right)\left(1+\sum_{j=1}^{N} \sum_{k=0}^{t_{j}-1}\left|\xi_{k}^{j}\right|^{t_{j}(1-\varepsilon) / k}\right),
\end{aligned}
$$

где $0<\varepsilon<1, f_{h}$ и $C$ измеримые по Борелю функиии, $|C(x, \xi)| \leqslant C_{M}$, если $|\xi| \leqslant M$. Тогда, если $n \geqslant t^{\prime}$, то $u_{j} \in W_{q}^{t_{j}}(\Omega$, lос) для любого конечного $q>1$, $u$, следовательно, $u_{j} \in C^{t_{j}-1, \delta}(\Omega), j=1, \ldots, N$, для любого $\delta$ из интервала $(0,1)$.

ДоказАтЕльство. Сначала, воспользовавшись результатом теоремы 2.1, покажем, что $u \in \stackrel{\circ}{H}_{a}^{t}(\Omega)$ с показателем $a>t^{\prime}-n / 2$.

Не ограничивая общности предположим, что область $\Omega$ содержит шар радиуса 2 с центром в точке $\mathscr{O}$. Рассмотрим концентрические шаровые слои $G_{\lambda}=$ $\{\lambda / 2<|x|<\lambda\}$. Из теоремы 3.1 и замечания 3.1 следует:

$$
\begin{gathered}
\sum_{j=1}^{N} \int_{G_{1}} \sum_{|\alpha|=1}^{t_{j}}\left|D^{\alpha} u_{j}(x)\right|^{2} d x \leqslant \psi\left(\max _{G_{1 / 2} \cup G_{1} \cup G_{2}}\left|u_{j}(x)\right|\right) \leqslant \psi(M), \\
M=\left\|u ; L_{\infty}(\Omega)\right\| .
\end{gathered}
$$

Здесь функция $\psi$ не зависит от областей $G_{1 / 2} \cup G_{1} \cup G_{2}, G_{1}$. Перейдем теперь от $G_{1}$ к произвольному слою $G_{\lambda}, \lambda<1$. Сделаем преобразование координат $x=\lambda y$. При этом области $G_{\lambda / 2}, G_{\lambda}, G_{2 \lambda}$ перейдут, соответственно, в $G_{1 / 2}$, $G_{1}, G_{2}$. Система (4.5) преобразуется следующим образом

$$
\begin{gathered}
\sum_{j=1}^{N} \sum_{\left|\alpha_{h j}\right|=t_{j}} a_{\alpha_{h j}}^{\prime}(y) \lambda^{-t_{j}} D^{\alpha_{h j}} u_{j}^{\prime}(y)=g_{h}\left(y, u_{j}^{\prime}, \ldots, D^{t_{j}-1} u_{j}^{\prime}\right)_{j=1, \ldots, N}, \quad h=1, \ldots, N, \\
\text { где } u_{j}^{\prime}(y)=u_{j}(\lambda y), a_{\alpha_{h j}}^{\prime}(y)=a_{\alpha_{h j}}(\lambda y), \\
g_{h}\left(y, u_{j}^{\prime}, \ldots, D^{t_{j}-1} u_{j}^{\prime}\right)=f_{h}\left(\lambda y, u_{j}^{\prime}, \ldots, \lambda^{-\left(t_{j}-1\right)} D^{t_{j}-1} u_{j}^{\prime}\right) .
\end{gathered}
$$


Умножим обе части уравнений на $\lambda^{2 m}$ :

$$
\begin{gathered}
\sum_{j=1}^{N} \sum_{\left|\alpha_{h j}\right|=t_{j}} a_{\alpha_{h j}}^{\prime}(y) \lambda^{2 m-t_{j}} D^{\alpha_{h j}} u_{j}^{\prime}(y)=\lambda^{2 m} g_{h}\left(y, u_{j}^{\prime}, \ldots, D^{t_{j}-1} u_{j}^{\prime}\right)_{j=1, \ldots, N}, \\
h=1, \ldots, N .
\end{gathered}
$$

Заметим, что соответствующий последней системе характеристический многочлен

$$
L_{1}(y, \xi)=\operatorname{det}\left(\lambda^{2 m-t_{j}} l_{h j}(y, \xi)\right)
$$

совпадает в соответствуюших точках с характеристическим многочленом $L(x, \xi)$. Принимая во внимание, что $\lambda<1$, из условия (4.6) имеем

$$
\begin{aligned}
& \sum_{h=1}^{N}\left|\lambda^{2 m} g_{h}\left(y, D^{\gamma} u_{j}^{\prime}(y)\right)\right|=\sum_{h=1}^{N}\left|\lambda^{2 m} f_{h}\left(\lambda y, \lambda^{-|\gamma|} D^{\gamma} u_{j}^{\prime}(y)\right)\right| \\
& \quad \leqslant \lambda^{2 m} \sum_{h=1}^{N}\left|f_{h}\left(x, D^{\gamma} u_{j}(x)\right)\right| \\
& \quad \leqslant \lambda^{2 m} C(x, u)\left(1+\sum_{j=1}^{N} \sum_{k=1}^{t_{j}-1}\left|D_{x}^{k} u_{j}\right|^{t_{j}(1-\varepsilon) / k}\right) \\
& \quad=\lambda^{2 m} C\left(\lambda y, u^{\prime}(y)\right)\left(1+\sum_{j=1}^{N} \sum_{k=1}^{t_{j}-1} \lambda^{-t_{j}(1-\varepsilon)}\left|D_{y}^{k} u_{j}^{\prime}(y)\right|^{t_{j}(1-\varepsilon) / k}\right) \\
& \quad \leqslant C\left(\lambda y, u^{\prime}(y)\right)\left(1+\sum_{j=1}^{N} \sum_{k=1}^{t_{j}-1}\left|D_{y}^{k} u_{j}^{\prime}(y)\right|^{t_{j}(1-\varepsilon) / k}\right) .
\end{aligned}
$$

Получили оценку (4.6) для функции $\lambda^{2 m} g\left(y, u_{1}^{\prime}, \ldots, D^{\gamma} u_{j}^{\prime}\right)$. Таким образом, в новых координатах $(y)$ справедлива оценка

$$
\left\|u_{j}^{\prime} ; W_{2}^{t_{j}}\left(G_{1}\right)\right\|^{2} \leqslant \psi(M), \quad 1 \leqslant j \leqslant N .
$$

Вернемся к координатам $(x)$ и умножим обе части неравенств, соответственно, на $\lambda^{2\left(a-t_{j}+n / 2\right)}$

$$
\int_{G_{\lambda}} \sum_{|\alpha| \leqslant t_{j}} \lambda^{2\left(a+|\alpha|-t_{j}\right)}\left|D^{\alpha} u_{j}(x)\right|^{2} d x \leqslant \psi(M) \lambda^{2\left(a-t_{j}+n / 2\right)} .
$$

Уменьшим $\lambda$ в левой части

$$
\int_{G_{\lambda}} \sum_{|\alpha| \leqslant t_{j}} r^{2\left(a+|\alpha|-t_{j}\right)}\left|D^{\alpha} u_{j}(x)\right|^{2} d x \leqslant C_{1} \psi(M) \lambda^{2\left(a-t_{j}+n / 2\right)} .
$$

Положим $\lambda=2^{-k}$ и просуммируем последнее неравенство по $0 \leqslant k \leqslant \infty$. Таким образом,

$$
\int_{\Omega} \sum_{|\alpha| \leqslant t_{j}} r^{2\left(a+|\alpha|-t_{j}\right)}\left|D^{\alpha} u_{j}(x)\right|^{2} d x<\infty
$$


при $a>t_{j}-n / 2$. Последнее условие выполнено для всех $j$ при $a>t^{\prime}-n / 2$, где $t^{\prime}=\max _{j} t_{j}$.

Аналогично докажем, что $f \in \stackrel{\circ}{H}_{a_{1}}^{0}, a_{1}>t^{\prime}(1-\varepsilon)-n / 2$. Действительно,

$$
\int_{G_{1}}\left|D^{k} u_{j}\right|^{2 t_{j}(1-\varepsilon) / k} d x \leqslant \psi_{1}(M) .
$$

Рассуждая тем же способом, что и выше, получим

$$
\int_{G_{\lambda}} \lambda^{2 t_{j}(1-\varepsilon) / k-n}\left|D^{k} u_{j}\right|^{2 t_{j}(1-\varepsilon) / k} d x \leqslant \psi_{1}(M) .
$$

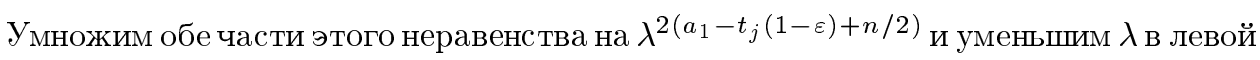
части

$$
\int_{G_{\lambda}} r^{2 a_{1}}\left|D^{k} u_{j}\right|^{2 t_{j}(1-\varepsilon) / k} d x \leqslant C_{2} \psi_{1}(M) \lambda^{2\left(a_{1}-t_{j}(1-\varepsilon)+n / 2\right)} .
$$

Положив $\lambda=2^{-i}$, просуммируем последнее неравенство по $0 \leqslant i \leqslant \infty$. Получим доказываемое.

Таким образом, на гладкость решения в точке $\mathscr{O}$ влияют полюсы $\mathscr{L}^{-1}(\lambda)$, лежащие в полосе

$$
t^{\prime}(1-\varepsilon)<a_{1}+n / 2<\operatorname{Im} \lambda<a+n / 2, \quad a+n / 2>t^{\prime} .
$$

Выберем $a$ и $a_{1}$ так, чтобы выполнялось условие $\sigma>a-a_{1}>0$. Тогда в указанной полосе есть только один полюс $\lambda=i t^{\prime}$, и по теореме 4.1 решение $u$ имеет вид

$$
\begin{aligned}
u_{j}(r, \omega)=\sum_{q=0}^{p_{1}-1} r^{t_{j}-t^{\prime}}(\log r)^{q} \psi_{q, j}(\omega)+w_{j}(r, \omega) \\
\quad=r^{t_{j}-t^{\prime}} \psi_{0, j}(\omega)+r^{t_{j}-t^{\prime}} \psi_{1, j}(\omega) \log r+w_{j}(r, \omega) .
\end{aligned}
$$

Покажем, что функция $w_{j} \in W_{p}^{t_{j}}(\Omega)$ с некоторым $p>1$, т.е.

$$
\sum_{0 \leqslant|\alpha| \leqslant t_{j}} \int_{\Omega}\left|D^{\alpha} w_{j}\right|^{p} d x<\infty, \quad j=1, \ldots, N .
$$

Рассмотрим каждое слагаемое в (4.8) в отдельности. Умножим и разделим подынтегральную функцию на $r^{s_{\alpha}^{j}}$ с некоторым $s_{\alpha}^{j}$ и применим неравенство Гёльдера с $1 / p_{1}+1 / q_{1}=1$

$$
\int_{\Omega}\left|D^{\alpha} w_{j}\right|^{p} d x<\left(\int_{\Omega}\left|D^{\alpha} w_{j}\right|^{p p_{1}} r_{\alpha}^{s_{\alpha}^{j}} p_{1} d x\right)^{1 / p_{1}}\left(\int_{\Omega} r^{-s_{\alpha}^{j} q_{1}} d x\right)^{1 / q_{1}} .
$$

Так как $w_{j} \in \stackrel{\circ}{H}_{a_{1}}^{t_{j}}$, первый сомножитель в правой части ограничен, если $p p_{1}=2$, $p_{1} s_{\alpha}^{j}=2\left(a_{1}+|\alpha|-t_{j}\right)$, а второй интеграл сходится при $n>s_{\alpha}^{j} q_{1}$. Рассмотрим цепочку неравенств

$$
n>\max _{\alpha, j} s_{\alpha}^{j} q_{1}=\max _{\alpha, j} s_{\alpha}^{j} \frac{2}{2-p}=\max _{\alpha, j} \frac{2 p\left(a_{1}+|\alpha|-t_{j}\right)}{2-p}>\frac{2 t^{\prime}(1-\varepsilon)-n}{2-p} .
$$


Следовательно, $p<n /\left(t^{\prime}(1-\varepsilon)\right)$, и, если $n \geqslant t^{\prime}, 0<\varepsilon<1$, то $w_{j} \in W_{p}^{t_{j}}(\Omega)$. На самом деле доказано, что $w_{j} \in W_{p_{j}}^{t_{j}}(\Omega)$, где $p_{j}<n /\left(t_{j}(1-\varepsilon)\right)$. Ввиду того, что $\varepsilon>0, n \geqslant t_{j}$, сушествует $p_{j}$, удовлетворяюшее неравенствам

$$
\frac{n}{t_{j}}<p_{j}<\frac{n}{t_{j}(1-\varepsilon)}
$$

При $n>t^{\prime}$ из условия $u_{j} \in \stackrel{\circ}{H^{t}} \underset{a}{j}, a>t^{\prime}-n / 2$, следует, что $u_{j} \in W_{p_{0}}^{t_{j}}$ с некоторым $p_{0}>1$. Отсюда по теореме 3.1 получаем утверждение теоремы.

Пусть теперь $n=t^{\prime}$. Так как $w_{j} \in W_{p_{j}}^{t_{j}}(\Omega), p_{j} t_{j}>n$, то по теореме вложения Соболева $w_{j}$ - ограниченные функции, и, следовательно, учитывая ограниченность $u_{j}$, имеем

$$
u_{j}(r, \omega)= \begin{cases}\psi_{0, j}(\omega)+w_{j}(r, \omega), & t_{j}=t^{\prime} \\ w_{j}(r, \omega), & t_{j} \neq t^{\prime}\end{cases}
$$

где $\psi_{0, j}(\omega) \in C^{\infty}(\Omega)$.

Таким образом, $u_{j} \in W_{p}^{t_{j}}(\Omega), p>1$, при $n=t^{\prime}$, и, следовательно, теорема доказана.

\section{Список литературы}

1. Бернштейн C. Н. Собрание сочинений. Т. 3. М.: АН СССР, 1960.

2. Nagumo $M$. On principally linear elliptic differential equations of second order // Osaka Math. J. 1954. V. 6. № 2. P. 207-229.

3. Ладыженская О. А., Уральцева $H$. Н. Линейные и квазилинейные уравнения эллиптического типа. М.: Наука, 1973.

4. Скрыпник И. В. Методы исследования нелинейных эллиптических граничных задач. М.: Наука, 1990.

5. Похожаев С.И. О квазилинейных эллиптических уравнениях высокого порядка // Дифференц. уравнения. 1981. Т. 17. №1. С. 115-121.

6. Аракчеев $C$. A. О некоторых классах линейных и квазилинейных уравнений с суммируемыми коэффициентами // Труды Всесоюзной конф. по уравнениям с частными производными, посвященной 75-летию И.Г. Петровского. М., 1978. С. 299-311.

7. Скрыпник И. В. Разрешимость и свойства решений нелинейных эллиптических уравнений // Итоги науки и техники. Современные проблемы математики. М.: ВИНИТИ, 1976. C. $131-237$.

8. Агмон C., Дуглис A., Ниренберг Л. Оценки вблизи границы решений эллиптических уравнений в частных производных при общих граничных условиях. 1. М.: ИЛ, 1962.

9. Agmon S., Douglis A., Nirenberg L. Estimates near the boundary for the solutions of elliptic differential equations, satisfying general boundary values. II // Comm. Pure Appl. Math. 1964. V. 17. №1. P. 35-92.

10. Galiardo E. Ulteriori proprieta di alcune classi di funsioni in piu variabili // Ricerche di Math. Napoli. 1959. V. 8. № 1. P. 24-51.

11. Nirenberg L. On elliptic partial differential equations // Annal. Scuola Norm. Sup. Piza. 1959. V. 13. P. $115-162$.

12. Мазья В. Г. Об устранимых особенностях ограниченњых решений квазилинейных эллиптических уравнений любого порядка // Зап. науч. семинаров ЛОМИ АН СССР. 1972. T. 27. №6. С. 116-130.

13. Соболев С. Л. Об одной теореме функционального анализа // Матем. сб. 1938. Т. 4. №3. C. 471-497. 
14. Йон $\Phi$. Плоские волны и сферические средние в применении к диффференциальньм уравнениям с частньми производньми. М.: ИЛ, 1958.

15. Calderon A.P., Zigmund A. On singular integrals // Amer. J. Math. 1956. V. 78. P. 289-309.

16. Агранович M. С., Вишик М.И. Эллиптические задачи с параметром и параболические задачи общего вида // УМН. 1964. Т. 19. №3. С. 289-309.

17. Кондратьев $B$. A. Краевые задачи для эллиптических уравнений в областях с коническими или угловыми точками // Труды ММО. 1967. Т. 16. С. 27-92.

18. Мазъя В.Г., Пламеневский Б. А. О коэффициентах в асимптотике решений эллиптических краевых задач в областях с коническими точками // Math. Nachr. 1977. V. 76. P. 29-60.

Московский государственньй

технический университет им. Н.Э. Баумана
Поступила в редакцию 16.02 .1995 